CS 2018.01.01.12

Bionatura Conference Series Vol 1. No 1. 2018

Publicación del IV CONGRESO INTERNACIONAL DE BIOTECNOLOGÍA Y

BIODIVERSIDAD IV CIBB Y XV FORO INTERNACIONAL BANANERO

INVESTIGATION / RESEARCH

Previos / Index /

\title{
Cadmio y arsénico en chocolate y arroz de Quito, Guayaquil y Cuenca - Ecuador
}

Cadmium and arsenic in chocolate and rice from Quito, Guayaquil and Cuenca Ecuador

Tierra, $W^{a}$, Otero, X.L. ${ }^{b}$, Ruales, $J^{a}{ }^{,}$Maldonado-Alvarado P. ${ }^{{ }^{*}}$

Disponible en: http://dx.doi.org/10.21931/RB/CS/2018.01.01.12

\section{RESUMEN}

El cadmio (Cd) y arsénico (As) son elementos presentes a lo largo de la cordillera de los Andes. Estos elementos representan un riesgo para la salud humana debido a las afectaciones para la salud que puede ocasionar la permanente exposición a estos elementos, los cuales pueden estar presentes alimentos como el chocolate y el arroz. El presente estudio tuvo como objetivo la evaluación de las concentraciones totales de arsénico y Cden chocolates, arroz, suelos y granos de cacao con el fin de identificar el grado de contaminación presente en estos alimentos. Las muestras de chocolate fueron recolectadas en Ecuador, en las provincias de Quito, Guayaquil y Cuenca, mientras que el arroz fue recolectado en la provincia del Guayaquil. Las muestras fueron procesadas y analizadas mediante la técnica de Espectroscopia de Absorción Atómica por Horno de Grafito (GFAAS). Las muestras de chocolate, únicamente una sobrepasó el límite máximo de $0,8 \mathrm{mg} / \mathrm{kg}$ para el Cd para consumo humano, mientras que las muestras de arroz no sobrepasaron el límite de máximo de $0,4 \mathrm{mg} / \mathrm{kg}$. El arsénico en las muestras de chocolate presentó concentraciones inferiores al límite de detección, mientras que las muestras de arroz no sobrepasaron el límite de 0,2 mg/kg para consumo humano. En los suelos de cacao, 
unas de veinte muestras de Guayaquil sobrepasaron el límite de $0,50 \mathrm{mg} / \mathrm{kg}$, mientras que, de las muestras, en particular de una hacienda aledaña a la ciudad de Quito, cinco de doce muestras sobrepasan el límite de $\mathrm{Cd}$, pero las muestras de cacao allí encontradas no sobrepasaron el límite de $0,8 \mathrm{mg} / \mathrm{kg}$. A partir de los resultados se concluye que no existe riesgo en las muestras de chocolate y arroz para la salud humana.

\section{ABSTRACT}

Cadmium and arsenic are elements present along the Andes mountains. These elements represent a risk to human health due to the health effects that may be caused by the permanent exposure to these elements, which may be present foods such as chocolate and rice. The objective of this study was to evaluate the total results of arsenic and cadmium in chocolates, rice, soils and cocoa beans in order to identify the degree of contamination present in these foods. Chocolate samples were collected in Quito, Guayaquil, Cuenca, provinces of Ecuador, while rice was collected in Guayaquil. The samples were processed and analyzed using the Graphite Furnace Atomic Absorption Spectroscopy (GFAAS) technique. One sample of the chocolate samples exceeded the maximum limit of $0.8 \mathrm{mg} / \mathrm{kg}$ for cadmium for human consumption, while the rice samples did not exceed the maximum limit of $0.4 \mathrm{mg} / \mathrm{kg}$. For arsenic, chocolate concentrations cannot be detected in the equipment, while rice did not exceed the $0.2 \mathrm{mg} / \mathrm{kg}$ limit for human consumption. In cocoa soils, one of twenty samples from Guayaquil exceeded the limit of $0.5 \mathrm{mg} / \mathrm{kg}$, while samples from a plantation adjacent to Quito city, five of twelve samples exceeded the limit of cadmium, but the samples of cocoa in this plantation did not exceed the limit of $0.8 \mathrm{mg} / \mathrm{kg}$. From the results it is concluded that there is no risk in chocolate and rice samples for human health.

\section{INTRODUCCIÓN}

Los metales pesados, como el arsénico y el Cd, se encuentran formando parte de rocas, suelos, sedimentos y aguas, alcanzando raras veces valores tóxicos. Sin embargo, la actividad humana ha liberado al medio natural grandes cantidades de estos elementos en formas móviles y biodisponibles que pueden llegar a representar una amenaza para la salud humana. ${ }^{1}$

\section{Cadmio}


El Cd presente en el suelo y aguas de medio no contaminados procede de la alteración de la roca, por lo cual su origen es natural. Además, este tiende a estar presente en depósitos aluviales y en rocas y emisiones volcánicas. El Cd en el suelo tiene alta movilidad en comparación a otros metales pesados. ${ }^{2}$. El enriquecimiento de $\mathrm{Cd}$ en el suelo se debe principalmente a origen antropogénico mediante la incorporación de productos químicos con trazas de $\mathrm{Cd}$ en su composición. ${ }^{3}$

El Cd tiene un tiempo aproximado de permanencia de 900 años, desafortunadamente, el $90 \%$ del Cd no se inmoviliza en el suelo. Una de las principales fuentes de Cden el suelo, son los fertilizantes a base de fósforo (54\%), por deposición aérea (41\%) y por la incorporación de abono de origen animal con presencia de $\mathrm{Cd} .{ }^{4}$ Los fertilizantes de fósforo, principalmente el superfosfato contiene altas concentraciones de metales pesados tales como $\mathrm{Cd}, \mathrm{Cu}$ y $\mathrm{Zn}$ como impureza. ${ }^{5}$ La incorporación paulatina de éstos elementos aumentaría la concentración de en el suelo y su posterior ingreso a los tejidos de las plantas, ya sea para consumo por parte del hombre o del ganado.

En el organismo, el Cd puede tener un tiempo de residencia por un periodo de 10 30 años, por lo que su excreción es lenta. Sin embargo, la exposición continua a este elemento causa efectos negativos a la salud de la persona, principalmente en los riñones. Estos órganos son los que acumulan mayor cantidad de $\mathrm{Cd}$ en el cuerpo humano. ${ }^{6} \mathrm{~A}$ intoxicación por cadmio en el organismo causa nauseas, vómito, edema pulmonar y principalmente deficiencia renal, cáncer de próstata. En países como Japón, la toxicidad por Cd produce osteomalacia, la cual es conocida como "Itai-itai". ${ }^{7}$ La ingesta máxima de Cd por día no debe sobrepasar de 0,0004 mg $\mathrm{Cd} / \mathrm{kg} /$ día. ${ }^{8}$

Ecuador es el primer productor de cacao fino de aroma, sin embargo, existen reportes sobre el alto contenido de este metal pesado. Las concentraciones incluso han sobrepasado el límite establecido por la Unión Europea. ${ }^{9}$ En un informe previo realizado por el Departamento de Ciencia de Alimentos y Biotecnología de la EPN, se identificó que el Cd principalmente está presente a profundidades de 0 a $20 \mathrm{~cm}$ en el suelo de cacao, además que este elemento posee alta biodisponibilidad en el caso del cultivo de arroz. El límite de Cd va de 0,8 a $0,9 \mathrm{mg} / \mathrm{kg}$ de chocolate, en función del contenido de cacao que tenga el chocolate. ${ }^{10}$ En Perú, se realizaron cuantificaciones de los niveles de As y Cd en chocolates de marcas comerciales. En ese estudio, las concentraciones de Cd variaron de 0,21 a $0,57 \mathrm{mg} / \mathrm{kg}$. No se registró contaminación ya que están por debajo de los límites permitidos. ${ }^{11} \mathrm{Sin}$ embargo, en un estudio realizado en Colombia, en chocolates con diferentes 
porcentajes de cacao se reportaron concentraciones elevadas de $\mathrm{Cd}$, con un valor promedio de $4,047 \mathrm{mg} / \mathrm{kg}$, muy por encima de los límites de 0,8 a $0,9 \mathrm{mg} / \mathrm{kg}$.

En la ciudad de Cuenca, se realizó un estudio para cuantificar los niveles de Cdde marcas de arroz blanco e integral, todas las muestras registraron valores menores a $0,05 \mathrm{mg} / \mathrm{kg}$, estos valores están por debajo del límite de $0,5 \mathrm{mg} / \mathrm{kg} .{ }^{10}$

\section{Arsénico}

El arroz es una buena fuente de macro y micro elementos necesarios en la dieta alimenticia del hombre. En su estado sin pulir (arroz integral), el arroz aporta con carbohidratos, proteína, minerales (zinc, cobre, potasio, entre otros), fibra, además es bajo en grasa. ${ }^{12}$ Sin embargo, es el alimento de mayor riesgo por contaminación por As en el mundo, ya que en comparación a otros cereales como el trigo la cebada éste puede acumular hasta 20 veces más As en los tejidos de la planta. ${ }^{13}$ Las condiciones de inundación a las que se desarrolla con mayor facilidad, trae consigo mayores rendimientos, permite el control de plagas, etc., permite la fácil disponibilidad tanto de As y Cd y su posterior ingreso y acumulación en los tejidos de la planta, incluido los granos de arroz. Ecuador es uno de los mayores consumidores de arroz en América, con un promedio de $129 \mathrm{~g}$ /día/persona, por lo que la exposición a estos metales es permanente. ${ }^{14}$

Sin embargo, se ha evidenciado contaminación en agua y suelo por As a lo largo de la Cordillera de los Andes en países como Perú, Chile, Bolivia y Argentina. ${ }^{15}$ Las altas temperaturas presentes en emisiones volcánicas liberan grandes cantidades de As, ya que este elemento es móvil a estas condiciones térmicas. Las emisiones volcánicas además arrojan cenizas que tienden a aumentar la cantidad de As en cuerpos de aguas.

El riesgo de toxicidad por ingestión de As es alto debido a su rápida absorción en el intestino. ${ }^{16}$ lo que desencadena en problemas de salud como queratosis, ${ }^{17}$ desarrollo de tumores malignos en la piel; aumento del riesgo de cáncer en los pulmones de personas fumadoras ${ }^{18}$, entre otros. Cabe indicar que la ingesta máxima de As no debe sobrepasar de 0,0003 mg As/kg/día. ${ }^{19}$

En Perú, se registraron concentraciones de As que oscilan entre 0,21 y $0,47 \mathrm{mg} / \mathrm{kg}$ en marcas comerciales de chocolates. ${ }^{11}$ Adicionalmente, en Ecuador se registraron concentraciones de As en arroz blanco de $0,067 \mathrm{mg} / \mathrm{kg}$, estos valores están por 
debajo del límite establecido de $0,2 \mathrm{mg} / \mathrm{kg} .{ }^{14}$

Este proyecto tuvo como objetivo la caracterización de metales pesados que pudieran representar un riesgo para la población, especialmente la infantil en las provincias de Quito, Guayaquil y Cuenca. Como productos de análisis se tomaron en cuenta a chocolates y arroz; matrices en las cuales se cuantificará el grado de contaminación por Cd y As.

\section{MATERIALES Y MÉTODOS}

Un análisis de riesgos fue realizado con el fin de identificar los alimentos de mayor consumo dentro de los grupos vulnerables de la población ecuatoriana. Dentro de la población vulnerable se consideró como grupo prioritario a los niños menores de 12 años, en los cuales, la ingesta de productos contaminados con metales pesados puede afectar negativamente su salud y su desarrollo físico e intelectual.

Se escogieron las marcas comerciales de chocolates de mayor consumo con el fin de evaluar la concentración de Cd y As. Se analizaron 47 muestras de chocolate de Quito, 10 de Guayaquil y 48 de Cuenca, además de 15 muestras de arroz pulido de la ciudad de Guayaquil, ya que se encuentra en la provincia de mayor producción de arroz a nivel nacional.

Para la cuantificación de $\mathrm{Cd}$ en chocolate, las muestras fueron molidas en un molino de aspas de acero inoxidable. Para la disgregación ácida, 0,5 g de muestras de chocolate fue tratada con $9 \mathrm{~mL}$ de $\mathrm{HNO}_{3}$ y $1 \mathrm{~mL}$ de $\mathrm{H}_{2} \mathrm{O}_{2}$. Las digestiones fueron realizadas en un horno microondas Milestone ETHOS - UP. Las muestras digeridas fueron aforadas a un volumen de $50 \mathrm{~mL}$ con agua miliQ.

$\mathrm{Al}$ igual que el chocolate, las muestras de arroz fueron molidas y tratadas mediante una digestión ácida: $0,5 \mathrm{~g}$ de muestra fue digerida con $5 \mathrm{~mL}$ de $\mathrm{HNO}, 5 \mathrm{~mL}$ de agua miliQ y $1 \mathrm{~mL}$ de $\mathrm{H}_{2} \mathrm{O}_{2}$. Finalmente, las muestras fueron aforadas a $25 \mathrm{~mL}$ con agua miliQ.

Muestras de suelos y granos de cacao fueron recolectados en fincas aledañas a las ciudades de Quito y Guayaquil. Se tomarán 20 muestras de suelos de cultivo a una profundidad de $20 \mathrm{~cm}$, la cual corresponde a la cara arable del suelo.

Adicionalmente, muestras mazorcas de cacao del lugar donde fueron obtenidas las muestras de suelo fueron obtenidas. Estas muestras serán almacenadas en bolsas Ziploc y almacenadas en un recipiente en refrigeración hasta su llegada al 
laboratorio. Las muestras fueron secadas, molidas y almacenada.

Las muestras de suelos fueron tratadas con una mezcla de $9 \mathrm{~mL}$ de HNO3 (65 \%), 3 $\mathrm{mL}$ de $\mathrm{HCl}(37 \%)$ y $1 \mathrm{~mL}$ de $\mathrm{H}_{2} \mathrm{O}_{2}(30 \%)$, disgregadas en el horno microondas y aforadas a $25 \mathrm{~mL}$ previo a su análisis por horno de grafito.

Las muestras de cacao fueron secadas, molidas y disgregadas en el horno microondas de la misma manera en que fueron tratadas las muestras de chocolates.

Las especificaciones para el análisis de Cd y As por Horno de Grafito se especifican en la Tabla 1.

\begin{tabular}{|l|l|l|}
\hline Parámetro & \multicolumn{2}{l}{ As } \\
\hline Modificadores & $\begin{array}{l}0,005 \mathrm{mg} \mathrm{Pd}+0,003 \\
\mathrm{mg} \mathrm{Mg(NO3)}\end{array}$ & $\begin{array}{l}0,05 \mathrm{mg} \mathrm{NH} 4 \mathrm{H} 2 \mathrm{PO} 4+0,003 \\
\mathrm{mg} \mathrm{Mg}(\mathrm{NO} 3)\end{array}$ \\
\hline Longitud de onda & $193,70 \mathrm{~nm}$ & $228,80 \mathrm{~nm}$ \\
\hline $\begin{array}{l}\text { Temperatura de } \\
\text { pirólisis }\end{array}$ & $1200{ }^{\circ} \mathrm{C}$ & $500{ }^{\circ} \mathrm{C}$ \\
\hline $\begin{array}{l}\text { Temperatura de } \\
\text { atomización }\end{array}$ & $2000^{\circ} \mathrm{C}$ & $1500^{\circ} \mathrm{C}$ \\
\hline
\end{tabular}

Tabla 1. Condiciones a emplear para el análisis de Cd y As en Horno de grafito

La cuantificación de las concentraciones de Cd en las muestras de arroz y chocolate fueron realizadas mediante Espectrometría de Absorción Atómica con acople de Horno De Grafito (GFAAS).

Para la elaboración de la curva de calibración se prepararon dos soluciones estándar de 10 y $2 \mu \mathrm{g} / \mathrm{L}^{\text {a }}$ partir de una solución estándar de $1000 \mathrm{mg} / \mathrm{L}$. En el programa Syngistix for AA se configuró la preparación de la curva de calibración con concentraciones de $0,1,0,5,1,0,5,0$ y $10 \mathrm{ug} / \mathrm{L}$. La solución Matriz - modificadora fue elaborada mediante la preparación de una solución al 1,0\% de $\mathrm{NH}_{4} \mathrm{H}_{2} \mathrm{PO}_{4} \mathrm{y}$ $0,06 \%$ de $\mathrm{Mg}\left(\mathrm{NO}_{3}\right)$ en un volumen de $1,2 \mathrm{~mL}$. Adicionalmente el blanco de calibración fue preparado con agua miliQ $+\mathrm{HNO}_{3}$ concentrado para obtener una solución al 0,1 \% de $\mathrm{HNO}_{3}$. 
Para determinar la validez del método empleado para el tratamiento de las muestras se emplearon los materiales de referencia: Baking chocolate (NIST SRM 2384), rice flour (NIST SRM 1568b) y San Joaquin soil (NIST SRM 2709a)

A fin de complementar este estudio se cuantificó el Factor de Bioacumulación (FBC). Este indicador permite evaluar la capacidad de las plantas para acumular metales pesados en sus tejidos: Si FCB es mayor a 1, existe mayor probabilidad de bioacumulación de $\mathrm{Cd}$ en un tejido. ${ }^{20}$

\section{RESULTADOS}

En la Tabla 2 se indican los resultados con las concentraciones totales de Cd y As en chocolate. Se obtuvo un porcentaje de recuperación de $\mathrm{Cd}$ en el material de referencia de 108,3\%. No fue posible cuantificar para el As en el chocolate, ya que el material de referencia no posee As cuantificable en su composición.

\begin{tabular}{|l|l|l|}
\hline \multicolumn{1}{|c}{ Ciudad } & Cd $(\mathrm{mg} / \mathrm{kg})$ & As $(\mathrm{mg} / \mathrm{kg})$ \\
\hline Quito $(\mathbf{n = 4 7 )}$ & $0,303 \pm 0,220$ & $0,013 \pm 0,037$ \\
& $(0.014-0,766)$ & $\left(\mathrm{N} . \mathrm{D}^{\mathrm{a}}-0,243\right)$ \\
\hline Guayaquil $(\mathbf{n}=\mathbf{1 0})$ & $0,193 \pm 0,183$ & $0,036 \pm 0,063$ \\
& $(0,004-0,602)$ & $(\mathrm{N} . \mathrm{D}-0,194)$ \\
\hline Cuenca $(\mathbf{n}=\mathbf{4 8})$ & $0,261 \pm 0,197$ & $0,026 \pm 0,059$ \\
& $(0.024-0.895)$ & $(\mathrm{N} . \mathrm{D}-0,288)$ \\
\hline
\end{tabular}

${ }^{a}$ N.D: No detectable.

Tabla 2. Concentraciones totales de $\mathrm{Cd}$ y As en chocolate

Los valores de las concentraciones de As en arroz $(n=15)$ es de $0,057 \pm 0,022$ $\mathrm{mg} / \mathrm{kg}$ Las concentraciones oscilaron entre 0,014 y $0,100 \mathrm{mg} / \mathrm{kg}$. Estos valores no superan la concentración máxima de As en arroz de $0.200 \mathrm{mg} / \mathrm{kg}$ (10), mientras que la concentración de $\mathrm{Cd}$ fue de $0,060 \pm 0,038 \mathrm{mg} / \mathrm{kg}$, y oscilaron entre 0,011 y 0,124 $\mathrm{mg} / \mathrm{kg}$, estos valores están por debajo de la concentración máxima de $0,500 \mathrm{mg} / \mathrm{kg}$. 
EL porcentaje de recuperación para Cd en arroz fue de 101,8 \% y el del As en de $94,1 \%$.

En la Tabla 3 se indican las concentraciones de Cd y As en suelos de cultivo de cacao en las ciudades de Quito y Guayaquil.

Para las muestras de suelo, se obtuvo un porcentaje de recuperación de 120,15\% para $\mathrm{Cd}$, mientras que para As se obtuvo un porcentaje de 161,8\%.

\begin{tabular}{|l|c|l|l|}
\hline \multicolumn{1}{|c|}{ Matriz } & \multicolumn{1}{c}{ Ciudad } & Cd $(\mathrm{mg} / \mathrm{kg})$ & As $(\mathrm{mg} / \mathrm{kg})$ \\
\hline \multirow{3}{*}{ Suelos de cacao } & Quito $(\mathrm{n}=12)$ & $0,051 \pm 0,014$ & $1,248 \pm 0,287$ \\
& & $(0,032-0,089)$ & $(0,686-1,731)$ \\
\cline { 2 - 4 } & Guayaquil $(\mathrm{n}=20)$ & $0,187 \pm 0,141$ & $5,492 \pm 1,283$ \\
& & $(0,014-0,642)$ & $(2,263-7,961)$ \\
\hline \multirow{2}{*}{ Granos de cacao } & Quito $(\mathrm{n}=12)$ & $0,489 \pm 0,143$ & N.D \\
& & $(0,218-0,684)$ & \\
\cline { 2 - 4 } & Guayaquil $(\mathrm{n}=20)$ & $0,648 \pm 0,089$ & N.D \\
& & $(0,498-0,804)$ & \\
\hline
\end{tabular}

Tabla 3. Concentraciones de Cd y As en suelos y granos de cacao.

\section{DISCUSIÓN}

Las muestras de chocolate poseen concentraciones variables $\mathrm{Cd}$ en su composición (Tabla 1).

Con estos valores, existe evidencia de bioacumulación de Cd en el grano de cacao $(\mathrm{FBC}=5,07 \pm 4,34)$. En consecuencia, existe alta capacidad de bioacumulación de $\mathrm{Cd}$ en el fruto de la planta de cacao. ${ }^{21}$

Cabe indicar que en el estudio no se encontró relación entre el contenido de $\mathrm{Cd}$ en el suelo y el contenido en el grano de cacao. Sin embargo, se encontró que el Cd en el grano es superior a la concentración de $\mathrm{Cd}$ en el suelo de cultivo, lo que indica la paulatina acumulación de este metal en el grano, aunque existan bajos contenidos presentes en el suelo de cultivo.

En un estudio realizado por Chávez, et al ${ }^{21}$, indica que el Cd que puede ser fácilmente absorbido por la planta tiene una correlación negativa con $\mathrm{pH}$, lo que 
indica que, a valores bajos de $\mathrm{pH}$, la concentración de Cd que puede existir en los tejidos de la planta de cacao aumenta. ${ }^{22}$ En este estudio, los valores de $\mathrm{pH}$ oscilan entre 6,7 y 8,3, lo que provocaría la inmovilización de $\mathrm{Cd}$ en el suelo y, en consecuencia, concentraciones que no representan un riesgo para la salud.

Lo mencionado anteriormente se confirma con las concentraciones de $\mathrm{Cd}$ en las marcas comerciales, ya que se detectó únicamente una muestra $(0,89 \mathrm{mg} / \mathrm{kg})$ que supera el límite de $0,8 \mathrm{mg} / \mathrm{kg}$ establecido por la OMS (10). Aunque el As está presente en las muestras de suelos de cacao, su disponibilidad se ve limitada principalmente por las condiciones a las que se desarrolla el cacao, ya que, a diferencia del arroz, el cacao no tiene que estar inundado para poderse desarrollar. La inundación del suelo reduce principalmente el potencial Redox (Eh), lo que aumenta la disponibilidad y absorción de As, principalmente en los cultivos de arroz inundado. 23

En los resultados obtenidos en las muestras de arroz, las muestras de arroz pulido no sobrepasan los límites de $0,4 \mathrm{mg} / \mathrm{kg}$ en $\mathrm{Cd}$ y de $0,2 \mathrm{mg} / \mathrm{kg}$ en As, establecido por la OMS. ${ }^{10}$

La mayor parte de minerales, así como de metales pesados se concentran en la parte externa del grano de arroz, conocida como salvado. Durante el proceso de pulido, se remueve la mayor parte de esta capa, lo que reduce el valor nutritivo del arroz, así como el contenido de metales pesados del grano de arroz, ${ }^{24}$ en consecuencia, se reduce el riesgo por toxicidad.

\section{CONCLUSIONES}

El análisis de metales pesados, particularmente $\mathrm{As} \mathrm{y} \mathrm{Cd}$, en los alimentos estudiados, reveló niveles de contaminación por debajo de los límites máximos permitidos, salvo en ciertas excepciones. Sin embargo, estudios más específicos de especiación de estos metales (ej. determinación de inorgánicas como arsenato y arsenito u orgánicas como el caso del ácido dimetilarsénico o monometilarsónico) podrían revelar patrones diferentes de toxicidad lo cual también se relaciona con el entorno de desarrollo agrícola y con el metabolismo después de la ingesta de alimentos contaminados.

La exposición crónica a metales pesados mediante la ingesta frecuente de alimentos contaminados, incluso a niveles por debajo de los límites máximos permitidos, puede desencadenar en complicaciones en la salud a mediano y largo plazo, debido 
a la acumulación paulatina de metales pesados en los tejidos del cuerpo humano.

\section{AGRADECIMIENTOS}

Este trabajo forma parte del proyecto "Fortalecimiento de la inocuidad alimentaria en el Ecuador: Estudio de los contaminantes microbianos, micotóxicos y de metales pesados en alimentos y establecimiento de un sistema nacional de capacitación en línea", financiado por la Red CEDIA y la Escuela Politécnica Nacional por el proyecto CEPRA XI-2017-02. Agradecemos al Departamento de Ciencia de Alimentos y Biotecnología por su colaboración con los equipos necesarios para las cuantificaciones de metales pesados.

\section{REFERENCIAS}

1. Otero XL, Huerta-Diaz MA, Macías F. Heavy metal geochemistry of saltmarsh soils from the Ría of Ortigueira (mafic and ultramafic areas, NW Iberian Peninsula). Environ Pollut [Internet]. 2000 Nov [cited 2018 Dec 19];110(2):28596. Available from: http://linkinghub.elsevier.com/retrieve/pii/S0269749199003024

2. Beesley L, Moreno-Jiménez E, Clemente R, Lepp N, Dickinson N. Mobility of arsenic, cadmium and zinc in a multi-element contaminated soil profile assessed by in-situ soil pore water sampling, column leaching and sequential extraction. Environ Pollut [Internet]. Elsevier; 2010 Jan 1 [cited 2018 Dec 16];158(1):155-60. Available from:

https://www.sciencedirect.com/science/article/pii/S0269749109003595

3. Gramlich A, Tandy S, Gauggel C, López M, Perla D, Gonzalez V, et al. Soil cadmium uptake by cocoa in Honduras. Sci Total Environ [Internet]. 2018 Jan [cited 2018 Jan 10];612:370-8. Available from: http://linkinghub.elsevier.com/retrieve/pii/S0048969717321411

4. Pérez P, Azcona M. Los efectos del cadmio en la salud. Rev Esp Méd Quir Vol [Internet]. 2012 [cited 2018 Jan 8];17(3). Available from: http://www.medigraphic.com/pdfs/quirurgicas/rmq-2012/rmq123i.pdf

5. Gimeno-García E, Andreu V, Boluda R. Heavy metals incidence in the application of inorganic fertilizers and pesticides to rice farming soils. Environ Pollut [Internet]. 1996 [cited 2017 Sep 28];92(1):19-25. Available from: http://linkinghub.elsevier.com/retrieve/pii/0269749195000909 
6. Nawrot T, Plusquin M, Hogervorst J, Roels HA, Celis H, Thijs L, et al. Environmental exposure to cadmium and risk of cancer: a prospective populationbased study. Lancet Oncol [Internet]. 2006 Feb [cited 2018 Jan 10];7(2):119-26. Available from: http://linkinghub.elsevier.com/retrieve/pii/S1470204506705459

7. Kawada T, Suzuki S. A review on the cadmium content of rice, daily cadmium intake, and accumulation in the kidneys. J Occup Health. 1998;40(4):264-9.

8. ATSDR. ToxGuide for Cadmium [Internet]. Atlanta; 2012 [cited 2018 Dec 18]. Available from: https://www.atsdr.cdc.gov/toxguides/toxguide-5.pdf

9. Rikolto en Sudamérica. Exportación de cacao ecuatoriano se ve amenazada por altos niveles de cadmio | Rikolto en Sudamérica [Internet]. 2015 [cited 2018 Jan 16]. Available from: https://sudamerica.rikolto.org/es/noticias/exportacion-decacao-ecuatoriano-se-ve-amenazada-por-altos-niveles-de-cadmio

10. WHO. 41st Session of the Codex Alimentarius Commission, Rome, 2-6 July 2018. Who [Internet]. World Health Organization; 2018 [cited 2018 Aug 31]; Available from: http://origin.who.int/foodsafety/areas_work/foodstandard/CAC41/en/index1.html

11. Velazque M. Determinación de arsénico, cadmio y plomo en barras de chocolate adquiridas en el centro comercial polvos azules de Lima Metropolitana en el periodo enero - febrero 2016 [Internet]. [Lima]: WIENER; 2017 [cited 2018 Dec 18]. Available from:

http://repositorio.uwiener.edu.pe/bitstream/handle/123456789/708/Titulo Velasque Leiva Miguel Angel.pdf?sequence $=1 \&$ isAllowed=y

12. Shi Z, Taylor AW, Wittert GA. Rice Intake, Weight Change and Metabolic Syndrome. In: Wheat and Rice in Disease Prevention and Health [Internet]. Elsevier; 2014 [cited 2018 Jan 11]. p. 323-31. Available from: http://linkinghub.elsevier.com/retrieve/pii/B9780124017160000246

13. Williams P, Villada A, Deacon C. Greatly enhanced arsenic shoot assimilation in rice leads to elevated grain levels compared to wheat and barley. Environmental [Internet]. 2007 [cited 2014 Oct 14];44(0):1-6. Available from: http://pubs.acs.org/doi/abs/10.1021/es070627i

14. Otero XL, Tierra W, Atiaga O, Guanoluisa D, Nunes LM, Ferreira TO, et al. 
Arsenic in rice agrosystems (water, soil and rice plants) in Guayas and Los Ríos provinces, Ecuador. Sci Total Environ [Internet]. Elsevier B.V.; 2016;573:778-87. Available from: http://dx.doi.org/10.1016/j.scitotenv.2016.08.162

15. Ormachea Muñoz M, Wern H, Johnsson F, Bhattacharya P, Sracek O, Thunvik R, et al. Geogenic arsenic and other trace elements in the shallow hydrogeologic system of Southern Poopó Basin, Bolivian Altiplano. J Hazard Mater [Internet]. 2013 Nov 15 [cited 2014 Oct 13];262:924-40. Available from: http://www.sciencedirect.com/science/article/pii/S0304389413004949

16. Matschullat J. Arsenic in the geosphere - a review. Sci Total Environ [Internet]. 2000 Apr [cited 2014 Oct 14];249(1-3):297-312. Available from: http://www.sciencedirect.com/science/article/pii/S0048969799005240

17. Mulware SJ. Trace elements and carcinogenicity: a subject in review. 3 Biotech [Internet]. 2012 Jun 10 [cited 2014 May 13];3(2):85-96. Available from: http://link.springer.com/10.1007/s13205-012-0072-6

18. Rossman T. Mechanism of arsenic carcinogenesis: an integrated approach. Mutat Res Mol Mech Mutagen [Internet]. 2003 Dec 10 [cited 2014 Sep 29];533(12):37-65. Available from:

http://www.sciencedirect.com/science/article/pii/S0027510703002082

19. ATSDR. ToxGuide for Arsenic [Internet]. Atlanta; 2007 [cited 2018 Dec 18]. Available from: https://www.atsdr.cdc.gov/toxguides/toxguide-2.pdf

20. Perlatti F, Ferreira TO, da Costa Roberto FA, Romero RE, Sartor LR, Otero $\mathrm{XL}$. Trace metal/metalloid concentrations in waste rock, soils and spontaneous plants in the surroundings of an abandoned mine in semi-arid NE-Brazil. Environ Earth Sci [Internet]. Springer Berlin Heidelberg; 2015 Sep 30 [cited 2018 Dec 19];74(6):5427-41. Available from: http://link.springer.com/10.1007/s12665-0154556-7

21. Chavez E, He ZL, Stoffella PJ, Mylavarapu RS, Li YC, Moyano B, et al. Concentration of cadmium in cacao beans and its relationship with soil cadmium in southern Ecuador. Sci Total Environ [Internet]. 2015 [cited 2018 Dec 16];533:20514. Available from: http://dx.doi.org/10.1016/j.scitotenv.2015.06.1060048-9697/

22. Bolan NS, Adriano DC, Mani PA, Duraisamy A. Immobilization and phytoavailability of cadmium in variable charge soils. II. Effect of lime addition. Plant Soil [Internet]. Kluwer Academic Publishers; 2003 [cited 2018 Dec 
16];251(2):187-98. Available from:

http://link.springer.com/10.1023/A:1023037706905

23. Yamaguchi N, Nakamura T, Dong D, Takahashi Y, Amachi S, Makino T. Arsenic release from flooded paddy soils is influenced by speciation, $\mathrm{Eh}, \mathrm{pH}$, and iron dissolution. Chemosphere [Internet]. Pergamon; 2011 May 1 [cited 2018 Dec 17];83(7):925-32. Available from:

https://www.sciencedirect.com/science/article/pii/S0045653511001901

24. Meharg AA, Lombi E, Williams PN, Scheckel KG, Feldmann J, Raab A, et al. Speciation and localization of arsenic in white and brown rice grains. Environ Sci Technol [Internet]. 2008 Feb 15 [cited 2018 Dec 16];42(4):1051-7. Available from: http://www.ncbi.nlm.nih.gov/pubmed/18351071

Tierra, $W^{a}$, Otero, X.L. ${ }^{b}$, Ruales, $J^{a}{ }^{,}$Maldonado-Alvarado P. ${ }^{{ }^{*}}$

${ }^{a}$ Escuela Politécnica Nacional, Departamento de Ciencia de Alimentos y Biotecnología, Quito, Quito, Ecuador.

${ }^{b}$ Departamento de Edafoloxía e Química Agrícola, Facultade de Bioloxía, Universidade de Santiago de Compostela, Campus Sur, 15782 Santiago de Compostela, España.

*Autor corresponsal: pedro.maldonado@epn.edu.ec 